\title{
Research on the Drug Allocation Model
}

\author{
Rui-fang Ji \\ School of North China Electric Power University, Baoding 071000, China; \\ 598561035@qq.com
}

Keywords: SIR model; the delivery systems; Ebola; the dynamic drug distribution model

\begin{abstract}
On the basis of studying to the SIR model, considering the spread of Ebola and the delivery systems, the drug allocation model is set up to optimize the distribution of drugs after the outbreak. Considering that the severity of the epidemic areas are different and their economic level is low, we establish the network of service station and the epidemic stricken area. Then the dynamic drug distribution model is built, which consists of two stages, in the early stage, drug is shortage. For the effective control of the epidemic, minimizing daily new infections number as the goal, and distribute drugs according to the situation in the disaster areas. In the later stage, drug is enough, with the goal of minimum cost, employing the knowledge of graph theory, the distribution is given. Finally, we apply the model to a case, and the model's rationality is verified by the results.
\end{abstract}

\section{Introduction}

Ebola virus disease (EVD) is a severe, often fatal illness in humans, with high infectivity, high pathogenicity and high mortality rate. It first appeared in 1976, and several outbreaks EVD have occurred in Western and Central Africa, with a case fatality rate of up to $90 \%{ }^{[1]}$. The EVD outbreak in Guinea in 2013 December has caught worldwide attention to the tremendous number of infections and deaths .Multinational epidemic prevention and medical forces have joined the fight against the epidemic in the action. And fortunately, after a long-term efforts, a new medicine, which can stop Ebola and cure patients whose disease is not advanced, is developed. In order to optimize the eradication of Ebola, or at least its current strain, we build a realistic model to optimize the distribution of drugs after the outbreak.

To study the spread of the EVD, various infectious disease models can be adopted. Among them, the improved SIR model is used most widely. Which originated from The SIR epidemic model $^{[2]}$ developed by Kermack and McKendrick with the kinetic method in 1972. This model provides a method for studying the development process of the disease, for revealing the regularity of epidemic, and for predicting the change trend.

In the establishment of delivery systems to the affected area transport drugs, many related transportation optimization method can be employed. One is the Graph theory. The Graph theory ${ }^{[3]}$ provides an optimal scheme for the road traffic transportation problem. It started in 1736, after Swiss mathematician Euler published L. solution of Konigsberg Seven Bridges problem articles.

Given that the outbreak often occurs in multiple areas ${ }^{[4]}$, establishing feasible delivery systems to deliver the corresponding drug to the affected area is necessary. From the collected data indicates, we discovered that the epidemic often outbreaks in the poor areas Africa, where the medical resources is limited and the supply of drug cannot meet the various epidemic disaster area demand in a short time. Only when the drugs production reach to a certain extent, can the demand of each epidemic stricken area of medicine be met. In the early time, drugs are deficient. To effectively control the spread of the epidemic, we regard the epidemic area total prevalence in all the fewest number as the goal, optimizing drug distribution of the epidemic area. In the later time, the drugs are availability.in view of the low local economic level, we regard the lowest transportation cost as the goal, using graph theory method for the service station to the area of drug the distribution plan. 


\section{The drug allocation optimization model}

\subsection{Analysis}

In the event of the Ebola virus outbreak, drug delivery is crucial for emergency demand response. Here, based on the spread of the Ebola disease and the extent of the disaster area, we study the optimal scheme for the situation with multiple distribution sites and disaster areas. First, according to the local transportation conditions and the topographic distribution of disaster area, the distribution location is determined. Then, given the main reasons for the outbreak in Africa: the low level of productivity and imperfect medical facilities[5], the supply of drugs are bound to affect the drug distribution scheme. Only when the speed of the drug production reaches a certain degree, can the demand of epidemic areas is met. So we have to consider the distribution in two stages, the former stage lacking drug and the stage with adequate drug.

\subsection{Build the model}

\subsubsection{Drug shortage period}

- Analysis

In the early time, the drug production ability is low, and the limited drug satisfy the demand. If just pour the medicine into part disasters areas, the epidemic of these places can be controlled effectively, while others may become out of control. Hence, to effectively control the spread of the epidemic, we regard the epidemic area total prevalence in all the fewest number as the goal, optimizing drug distribution of the epidemic area.

- Assumptions

Assume that the area's drug demand is proportional to the local cure rate

The number of the diagnosed is equal to the incidence

- Symbols and Definitions

\begin{tabular}{ll}
\hline Symbol & Definition \\
\hline$\Delta C_{t k}$ & the new onset of incidence number in the day i of the disaster $\mathrm{k}$ \\
$L_{t k}$ & the latency number in the day i of the disaster $\mathrm{k}$ \\
$C_{t h}$ & the latency number \\
$r_{k}$ & the ratio of total healers by the day $\mathrm{t}$ \\
$W_{t k}$ & the rug distribution of the disaster in the day $\mathrm{t}$ \\
$N_{t}$ & the total rug distribution in the day t \\
\hline
\end{tabular}

- Build the model

This is a resource optimization problem, at this stage, to more effectively control the whole epidemic situation, we regard the epidemic area total prevalence in all the fewest number as the goal. That is the goal is:

$$
\min \sum_{k=1}^{n} \Delta C_{t k}=l L_{t k}-r_{k} C_{t k}
$$

Seeing the goal, every area has its rug distribution demand, which is proportional to the local cure rate. Based on the assumption, we find that the distribution is also proportional to the local cure rate.

$$
w_{i k}=r_{k} \beta
$$

Among that, $\beta$ is a constant per day, and $\beta>0$.

Considering the rug is limited, but increase along with the time, assume the amount of the rug per day is $N_{t}$,

So, the optimization model is

$$
\sum_{k=1}^{n} w_{t k} \leq N_{t}
$$

$$
\text { s.t } \min \sum_{k=1}^{n} \Delta C_{t k}=l L_{t k}-r_{k} C_{t k}
$$




\subsubsection{Drug sufficient period}

$$
\left\{\begin{array}{l}
W_{t k}=r_{k} \beta \\
\sum_{k=1}^{n} w_{t k} \leq N_{t} \\
\beta>0 \\
r_{k} \leq 30 \%
\end{array}\right.
$$

- Analysis

In the later time, the drugs are availability.in view of the low local economic level, we regard the lowest transportation cost as the goal, using graph theory method for the service station to the area of drug the distribution plan.

- Assumptions:

$>$ Assume that the locations of the distribution centers also are previously known.

$>$ Assume that the affected areas are isolated from each other without population mobility when an epidemic strikes.

$>$ Assume that the rug throughput of each distribution area on behalf of its drug production speed the former day.

- Symbols and Definitions

\begin{tabular}{cl}
\hline Symbol & Definition \\
\hline$L_{\mathrm{ij}}$ & the distance between the destination $\mathrm{i}$ to the disaster $\mathrm{j}$ \\
$Q_{j}$ & the drug demand of the disaster $\mathrm{j}$ \\
$\lambda$ & the unit price transporting unit medicine in unit distance \\
$X_{\mathrm{i} j}$ & the drug quantity transport from the destination to the disaster $\mathrm{j}$ \\
$W_{i}$ & the drug quantity of the destination $\mathrm{j}$ \\
$\mathrm{Z}$ & the total transportation cost \\
\hline
\end{tabular}

- $\quad$ Build the model ${ }^{[6]}$

This is still a resource optimization problem, at this stage, drug is enough. Considering the actual local economy level is low, we aiming at maximum saving transportation cost, the goal is

$$
\min Z=\sum_{i=1}^{m} \sum_{j=1}^{n} \lambda L_{i j} X_{i j}
$$

The service station to the disaster area transportation demand for drugs sum equal to the area of drugs

$$
\sum_{i=1}^{m} X_{i j}=Q_{j}
$$

In real life, service stations' total number of export drugs of service station can't be more than the number of their drugs.

$$
\sum_{j=1}^{n} X_{i j} \leq w_{i}
$$

So, this optimization problem show as below:

$$
\begin{gathered}
\text { s.t } \quad \min Z=\sum_{i=1}^{m} \sum_{j=1}^{n} \lambda L_{i j} X_{i j} \\
\left\{\begin{array}{l}
\sum_{i=1}^{m} X_{i j}=Q_{j} \\
\sum_{j=1}^{n} X_{i j} \leq W_{i} \\
X_{i j} \geq 0
\end{array}\right.
\end{gathered}
$$




\section{Simulation of disease}

Now, there are five Ebola epidemic areas, two service station. When the disease outbreak, the total amount of drug of two service stations is 100, in which add 10 per day. The drug is enough at the 44th day. In this period of time, assume that there is no person die of disease in this area. The population is constant.

The distribution of known service stations and the epidemic areas show as below:

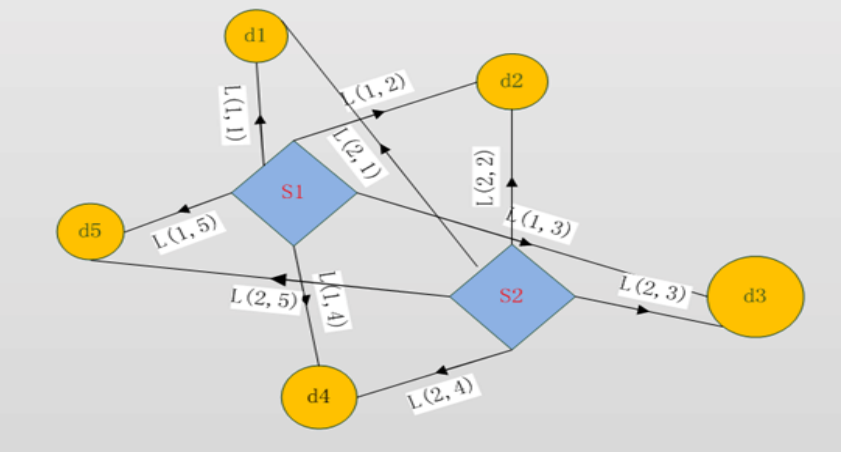

Fig 3.1 The distribution of known service stations and the epidemic areas

The population of the five epidemic areas $\{\mathrm{d} 1, \mathrm{~d} 2, \mathrm{~d} 3, \mathrm{~d} 4, \mathrm{~d} 5\}$ are $\{1100,1200,1300$, $1400,1250\}$ When lack of drugs, the area of drugs to be delivered is proportional to the cure rate, the ratio is 600.According to the epidemic situation prediction, the number of drugs of $\{\mathrm{d} 1, \mathrm{~d} 2$, d3, d4, d5 $\}$ is $\{374,408,442,743,695\}$ The distance between service stations and epidemic areas show as below:

Tab 3.1 The distance between service stations and epidemic areas

\begin{tabular}{llllll}
\hline$L_{\mathrm{i} j}$ & $\mathrm{~d} 1$ & $\mathrm{~d} 2$ & $\mathrm{~d} 3$ & $\mathrm{~d} 4$ & $\mathrm{~d} 5$ \\
\hline $\mathrm{S} 1$ & 10 & 15 & 40 & 16 & 9 \\
$\mathrm{~S} 2$ & 38 & 17 & 10 & 11 & 30 \\
\hline
\end{tabular}

Analysis: We can know from the question, 1-43 days after the outbreak is the drug-lack period, drug is enough from the 44th day on. Therefore, distribute drugs on the next day by the method of model in 2.2.1 and distribute drugs on the 45th day by the method of model in 2.2.2. The distribution show as below:

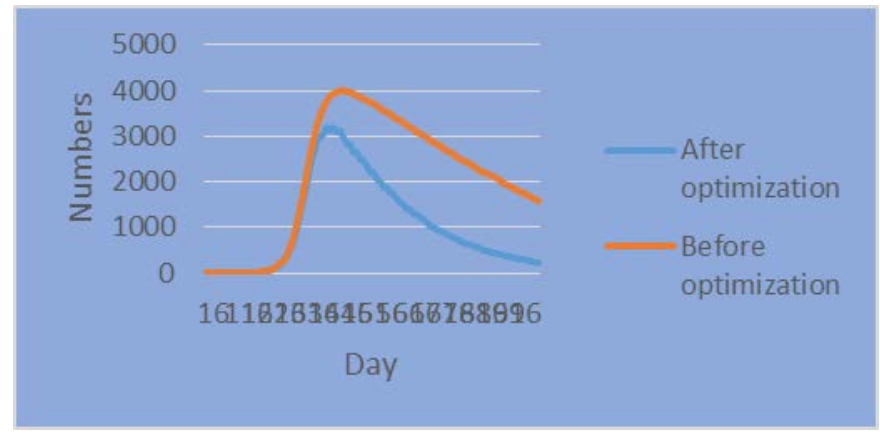

Fig 3.2 the curves of the total incidence before and after optimal drug distribution 6th day, analysis and build the model as below:

$$
\begin{aligned}
& \text { s.t } \min \sum_{k=1}^{5} \Delta C_{6 k}=l L_{6 k}-r_{k} C_{6 k} \\
& \left\{\begin{array}{c}
W_{6 k}=r_{k} \beta \\
\sum_{k=1}^{5} W_{6 k} \leq 150 \\
\beta=600 \\
r_{k} \leq 30 \%
\end{array}\right.
\end{aligned}
$$

Result: $w_{61}=12 w_{62}=18 w_{6 \mathrm{a}}=36 w_{64}=54 w_{65}=30$. That is to say the distribution of epidemic areas $\{d 1, d 2, d 3, d 4, d 5\}$ are $\{12,18,36,54,30\}$ on 6th day. 
45th day: The epidemic area of drug demand and the distance between service stations and epidemic areas are known, take them into model 3.2. To calculate the number of export drug to epidemic areas from each service station on 45th day, show as below:

Tab 3.2 the distribution on the $45^{\text {th }}$ day

\begin{tabular}{llllll}
\hline & $\mathrm{d} 1$ & $\mathrm{~d} 2$ & $\mathrm{~d} 3$ & $\mathrm{~d} 4$ & $\mathrm{~d} 5$ \\
\hline S1 & 374 & 408 & 0 & 107 & 442 \\
S2 & 0 & 0 & 743 & 588 & 0 \\
\hline
\end{tabular}

The cost of transportation on this day $Z=29448$, the export of service station S1 and S2 both are 1331, that is to say, the manufacture speed of each service station on 44th day both are 1331 .

\section{Summary}

The method of the model is differential equation model. This model conforms with the continuity of the epidemic spread, which has a strong scientific and rationality. This method and thinking is widely used in analyzing the transmission of infectious disease. When promoting the model to analysis other diseases, we only need modify some of parameters to make a quick analysis of the new epidemic.

\section{References}

[1] http://en.wikipedia.org/wiki/Ebola_virus_disease

[2] http://wenku.baidu.com/view/cbfaec0103d8ce2f00662373.html

[3] http://baike.sogou.com/v168387.htm

[4]http://baike.sogou.com/lemma/default/ShowLemmaDefault.e;jsessionid=1719E174904C5C14B0 B5EACD36695D4C.n2?sp=l76691561\&syn=2014\%E5\%B9\%B4\%E5\%9F\%83\%E5\%8D\%9A\%E6 \%8B\%89\%E7\%97\%85\%E6\%AF\%92\%E7\%96\%AB\%E6\%83\%85

[5] http://www.guokr.com/article/439133/

[6] Xie Jinxing, franci c. Optimization modeling and LINDO/LINGO software [M]. Beijing: tsinghua university, tsinghua university press, 2005. 